This is an electronic reprint of the original article. This reprint may differ from the original in pagination and typographic detail.

Author(s): Topic, Filip; Rissanen, Kari

Title: $\quad$ Systematic Construction of Ternary Cocrystals by Orthogonal and Robust Hydrogen and Halogen Bonds

Year: $\quad 2016$

Version:

Please cite the original version:

Topic, F., \& Rissanen, K. (2016). Systematic Construction of Ternary Cocrystals by Orthogonal and Robust Hydrogen and Halogen Bonds. Journal of the American Chemical Society, 138(20), 6610-6616. https://doi.org/10.1021/jacs.6b02854

All material supplied via JYX is protected by copyright and other intellectual property rights, and duplication or sale of all or part of any of the repository collections is not permitted, except that material may be duplicated by you for your research use or educational purposes in electronic or print form. You must obtain permission for any other use. Electronic or print copies may not be offered, whether for sale or otherwise to anyone who is not an authorised user. 


\title{
Systematic Construction of Ternary Co-crystals by Orthogo- nal and Robust Hydrogen and Halogen Bonds
}

\author{
Filip Topić and Kari Rissanen* \\ University of Jyvaskyla, Department of Chemistry, Nanoscience Center, P.O. Box 35, FI-40014 University of \\ Jyvaskyla, Finland. \\ Supporting Information Placeholder
}

\begin{abstract}
A carefully designed strategy is presented for construction of ternary co-crystals, based on the orthogonality of two supramolecular interaction modes: hydrogen bonding between crown ethers and thioureas and the halogen bonding between thioureas and perfluorohalocarbons. Tested on a set comprising two crown ethers, two thioureas and five halogen bond donors, the strategy resulted in a high, $75 \%$ success rate, with 15/20 component combinations yielding at least one co-crystal. Crystal structure analysis revealed the interplay between the hydrogen and halogen bonding motifs, also shedding light on the variables affecting their formation.
\end{abstract}

\section{INTRODUCTION}

In the last two decades, one of the intensively pursued research topics in crystal engineering has been that of co-crystals, ${ }^{1,2}$ although they have been known for much longer. ${ }^{3}$ However, this comes as no surprise, given their great potential as e.g. pharmaceutical, ${ }^{4-6}$ luminescent, ${ }^{7}$ photo-mechanical, $^{8}$ ferroelectric ${ }^{9}$ or energetic ${ }^{10,11}$ (explosive!) materials. From the very beginning, one of the challenges has been the rational design of strategies enabling deliberate and systematic construction of cocrystals. While this has largely been overcome in case of binary co-crystals (i.e. comprising two components), much fever strategies have been developed for successful construction of ternary and more complex co-crystals. The seminal paper, not only posing this challenge, but also offering the first examples of design-based hydrogen-bonded ternary molecular solids (co-crystal), was that of Aakeröy et al., ${ }^{12}$ wherein two different carboxylic acid molecules were co-crystallized with isonicotinamide. More specifically, the stronger of the two acids was postulated to bind to the pyridine moiety of isonicotinamide while the weaker acid binds to the amide moiety, yielding a discrete 1:1:1 ternary supermolecule, by exploiting the hierarchy ${ }^{13}$ of supramolecular synthons. ${ }^{14}$ The strategy was later shown to be a general one, using different components selected based on the same principles. ${ }^{15-17}$

A great body of work on the preparation of ternary ${ }^{18-22}$ and even quaternary ${ }^{23,24}$ co-crystals has been recently reported by Desiraju and co-workers. First, a shapebased approach was devised, where one component in a binary co-crystal can be partially replaced by a third component, $^{25}$ in such a way that the molecules participating in (stronger) hydrogen bonding are left in place, while the weakly interacting molecules of the same kind are substituted by similarly shapes ones. This was demonstrated through successful substitution of loosely bound 4,4'-bipyridine molecules in its co-crystals with 2or 5 -methylresorcinol by similarly shaped molecules. ${ }^{18}$ In what could be considered as an extension of this approach, robust synthons were identified in a series of crystal forms based on binary co-crystals comprising heterocyclic bases and polyhydroxyphenols. Upon the introduction of a third component, the more robust synthons persisted while the presumably less reliable ones incorporated the newly introduced third component instead, yielding ternary co-crystals. ${ }^{19,20}$ Remarkably, the same reasoning could be applied sequentially to afford quaternary $^{23,24}$ and even quinternary ${ }^{23}$ co-crystals. Finally, Desiraju and colleagues also reported the so far only strategy utilizing a combination of halogen $\left(\mathrm{C}-\mathrm{X} \cdots \mathrm{O}_{2} \mathrm{~N} ; \mathrm{X}\right.$ $=\mathrm{I}, \mathrm{Br}$ ) and hydrogen bonding (carboxylic acid-amide heterosynthon) to construct binary or ternary cocrystals, ${ }^{21}$ depending on the exact nature of the components bearing these functional groups. ${ }^{22}$

We have recently been interested in using concerted hydrogen and halogen bonding to construct sophisticated supramolecular assemblies both in solution and the solid state. A particularly efficient approach was adopted where $N$-alkylammonium resorcinarene halide (NARX), ${ }^{26,27}$ supramolecular species consisting of different cationic resorcinarene derivatives and hydrogen bonded halide anions, were used as halogen bond acceptors with a number of donors. All three components ( $N$-alkylammonium resorcinarene cations, halide anions and halogen bond donors) could be varied in an orthogonal manner to yield deep cavity cavitands ${ }^{28,29}$ and capsules, ${ }^{30}$ as well as various dumbbell-like and polymeric pseudocapsular architectures. ${ }^{31}$ Inspired by this, we set out to devise a strategy for assembling ternary cocrystals with distinct supramolecular role for each component which would, by virtue of the orthogonality of hydrogen and halogen bonds, also allow for tuning of the structure by changing one, two or all of the components while still successfully producing the ternary co-crystal.

One of the first questions one faces when designing such a strategy is evaluating its outcomes. When can a 
strategy be deemed successful? We propose the following criteria: First, as an obvious conditio sine qua non, it has to yield ternary co-crystals. Second, it should do so with a high supramolecular yield, ${ }^{32}$ and finally, the precise control of the stoichiometry and structure should be achieved.

In order to achieve all these goals, we adopted an entirely new approach. Arman et al. reported that the $\mathrm{N}-\mathrm{H} \cdots \mathrm{S}$ type hydrogen bonding between thiourea-type molecules is preserved in the structures of their $\mathrm{C}-\mathrm{I} \cdots \mathrm{S}$ halogen bonded co-crystals. ${ }^{33}$ Moreover, the set of cocrystals described consisted of three different thioureas and three different halogen bond donors, suggesting that the orthogonality of hydrogen and halogen bonding could be a general property of such systems. Later, Robertson et al. showed the halogen bonds between diiodine as the donor and tetramethylthiourea as the acceptor to be particularly robust in solution, which was ascribed to their pronounced charge-transfer character. ${ }^{34}$ Inspired by the former and encouraged by the latter, we decided to combine these approaches by introducing a third component which would engage in hydrogen bonding with thiourea without competing for the halogen bond donor, yielding ternary co-crystals by two parallel but orthogonal self-assembly processes. Here, crown ethers emerged as an unorthodox but promising choice (Scheme 1).

\section{Scheme 1. Motif combination}

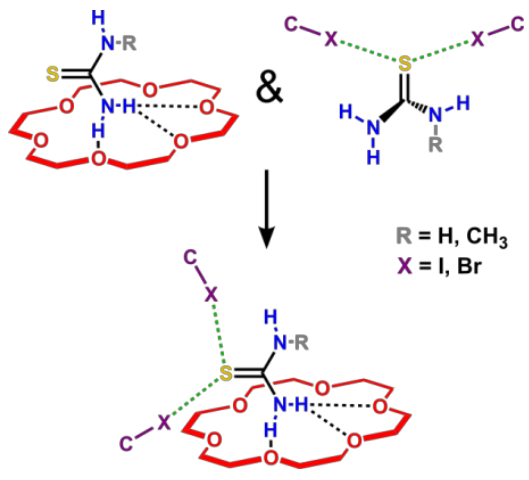

Possessing only ether oxygen atoms as possible acceptors, they were expected to reliably interact with thiourea NHR $(\mathrm{R}=\mathrm{H}$, alkyl) groups through multiple hydrogen bonds, thanks to the macrocyclic effect. ${ }^{35-37}$ At the same time, due to the high directionality of halogen bonds with respect to the donor, ${ }^{38}$ hypothetical formation of halogen bonds with crown ether would not benefit from such enhancement, thus being restricted to $\mathrm{C}-\mathrm{X} \cdots \mathrm{O}$ (ether) interactions, which we expected to be easily outcompeted by $\mathrm{C}-\mathrm{X} \cdots \mathrm{S}$ (thiourea) interactions with their more pronounced covalent character. ${ }^{34,39}$ Finally, as our design proved successful, these components will allow for easy expansion of the component set - a large number of thioureas, crown ethers of various sizes and perfluorinated halogen bond donors are commercially available, not to mention the easy synthetic accessibility of many more.

\section{RESULTS AND DISCUSSION}

Our starting component set (Scheme 2) thus comprised two crown ethers (A and $\mathbf{B}$ ), two different thiou- reas ( $\mathbf{C}$ and $\mathbf{D})$ and five different perfluorinated halogen bond donors, encompassing mono- and ditopic, aromatic and non-aromatic, linear and non-linear and iodine- and bromine-based ones $(\mathbf{1}$ - 5). The co-crystallization screening was initially conducted by simple solution crystallization, which was subsequently modulated where necessary to yield better diffraction quality crystals or a pure bulk phase (see SI).

Scheme 2. Components used
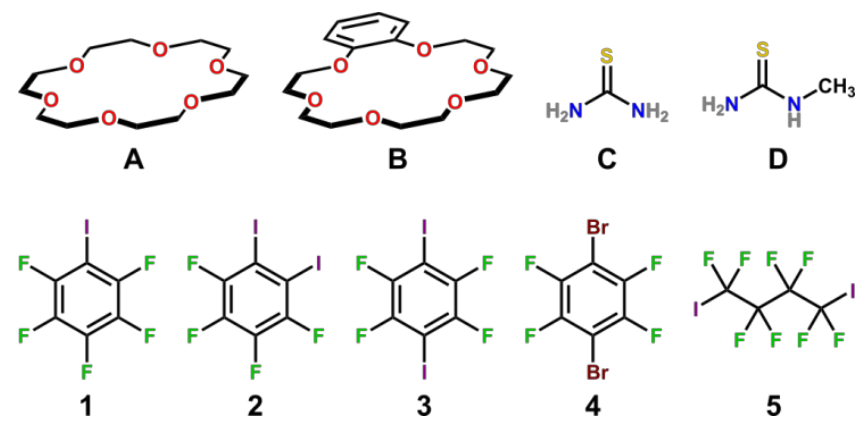

The results are summarized in Table 1.; Out of 20 possible $(2 \times 2 \times 5)$ combinations, 15 yielded at least one ternary co-crystal, thus giving a high supramolecular yield of $15 / 20=75 \%$. Taking into account the stoichiometric variations $\left(\mathbf{A} \cdot \mathbf{D} \cdot \mathbf{2}_{\mathbf{2}} / \mathbf{A} \cdot \mathbf{D}_{\mathbf{2}} \cdot \mathbf{2}_{\mathbf{2}}\right)$, polymorphism $(\alpha-$ B.C.1 $1_{2} / \beta-B \cdot C \cdot 1_{2}$ ) and the fact that some structures were determined at different temperatures, this resulted in a total of 21 different crystal structures (Tables S1a-S1e).

Table 1. The obtained ternary co-crystals

\begin{tabular}{|c|c|c|c|c|c|c|}
\hline & & 1 & 2 & 3 & 4 & 5 \\
\hline \multirow[b]{2}{*}{ A } & C & $A \cdot C \cdot 1_{2}$ & $A \cdot C_{2} \cdot 2_{2}$ & $A \cdot C_{2} \cdot 3_{2}$ & $A \cdot C_{2} \cdot 4_{2}$ & A.C. 5 \\
\hline & D & A.D. $\mathbf{1}_{2.2}$ & $\begin{array}{l}\text { A.D. } \mathbf{2}_{2} \\
\text { A.D. }{ }_{2} \cdot \mathbf{2}_{2}\end{array}$ & $A \cdot D_{2} \cdot \mathbf{3}_{2}$ & $A \cdot D \cdot 4_{2}$ & $A \cdot D \cdot 5$ \\
\hline \multirow[t]{2}{*}{ B } & C & $\begin{array}{l}\alpha-B \cdot C \cdot 1_{2} \\
\beta-B \cdot C \cdot 1_{2}\end{array}$ & $-{ }^{a}$ & $B \cdot C \cdot 3$ & $-{ }^{a}$ & B.C.5 \\
\hline & D & $-^{a}$ & $-{ }^{a}$ & $B \cdot D_{2} \cdot 3_{2}$ & $-{ }^{a}$ & B.D.5 \\
\hline
\end{tabular}

${ }^{a}$ No ternary phases were observed.

Given the expected orthogonality of hydrogen and halogen bonds in the ternary co-crystal structures, it should be possible to analyze the hydrogen and halogen bonding motifs independently. Focusing on the hydrogen bonding between $\mathbf{A} / \mathbf{B}$ and $\mathbf{C} / \mathbf{D}$, two binding stoichiometries can be observed, respectively $1: 1$ and $1: 2$, originating in three different binding motifs between them (Scheme 3). Whereas the motif III only appears in two polymorphs of B.C.1 $\mathbf{1}_{2}$ and can thus be considered somewhat exotic, the rest of the structures exhibit either motif I or II, which makes it worthwhile to explore the possible factors that steer the formation of one or the other. Fortunately, our component set was tailored to enable the comparative analysis of the behavior of each crown ether/thiourea combination in ternary co-crystals with up to 5 different halogen bond donors. 
Scheme 3. Hydrogen bond-mediated binding motifs between crown ethers and thioureas.
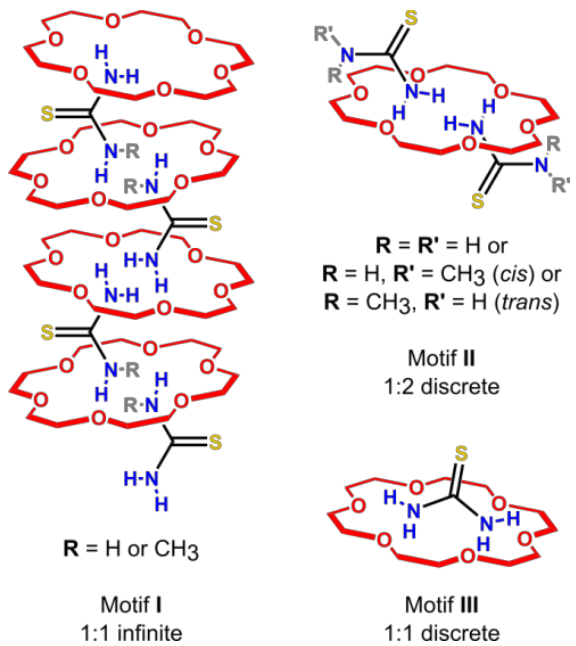

Individual hydrogen bonds are not marked as they vary between different occurrences of the same motif.

Thus, for the co-crystal subset with $\mathbf{A}$ and $\mathbf{C}$, motif II occurs in $3 / 5$ co-crystals $\left(\mathbf{A} \cdot \mathbf{C}_{2} \cdot \mathbf{2}_{2}, \mathbf{A} \cdot \mathbf{C}_{2} \cdot \mathbf{3}_{2}, \mathbf{A} \cdot \mathbf{C}_{2} \cdot \mathbf{4}_{2}\right)$, with the remaining two exhibiting motif I (Table 1; also vide infra). This might be correlated with the structure of previously described binary co-crystal $\mathbf{A} \cdot \mathbf{C}_{2},{ }^{35}$ which also shows motif II, indicating a slight preference for this binding motif, i.e. stoichiometry, with the combination of $\mathbf{A}$ and $\mathbf{C}$. Moreover, in the systems where II is present, with only one $\mathrm{NH}_{2}$ group fully engaged in hydrogen bonding with $\mathbf{A}$, the other $\mathrm{NH}_{2}$ group is able to facilitate aggregation of $\mathbf{A} \cdot \mathbf{C}_{2}$ moieties by forming further hydrogen bonds. This is indeed the case for $\mathbf{A} \cdot \mathbf{C}_{2} \cdot \mathbf{3}_{2}$ and $\mathbf{A} \cdot \mathbf{C}_{2} \cdot \mathbf{4}_{2}$, where 2D sheets are formed through $\mathrm{N}-\mathrm{H} \cdots \mathrm{S}$ hydrogen bonds between $\mathbf{A} \cdot \mathbf{C}_{2}$ moieties (Figure 1a). It thus comes as no surprise that the two co-crystals are in fact isomorphous, with the sheets interlinked through halogen bonding with $\mathbf{3}$ or $\mathbf{4}$, respectively, which also form stacks between the sheets (Figure 1b,1c). Contrasting that is $\mathbf{A} \cdot \mathbf{C}_{2} \cdot \mathbf{2}_{2}$, where the $\mathbf{A} \cdot \mathbf{C}_{2}$ moieties are instead linked into chains by $\mathrm{C}-1 \cdots S$ halogen bonding with 2 (Figure 2). This reveals a clear hydrogen bonding hierarchy in these systems, where the recognition of $\mathbf{C}$ by $\mathbf{A}$ through multiple $\mathrm{N}-\mathrm{H} \cdots \mathrm{O}$ hydrogen bonds is much more reliable than the $\mathrm{N}-\mathrm{H} \cdots \mathrm{S}$ hydrogen bonding, which can be overruled even by $\mathrm{C}-\mathrm{l} \cdots \mathrm{S}$ halogen bonding.

Still, judging from the relevant ternary co-crystal structures described here as well as the structure of their binary co-crystal, motif II is clearly preferred with $\mathbf{A}$ and C. The combination of $\mathbf{A}$ and $\mathbf{D}$ shows significantly different trends, with the preference for motif I starting from the previously described binary co-crystal $\mathbf{A} \cdot \mathbf{D}^{36}$ and also observed in 4/6 ternary co-crystals reported here. With the potential of halogen bond (XB) donors 1 and 5 for facilitating the formation of motif I already established, its reappearance in $\mathbf{A} \cdot \mathbf{D} \cdot \mathbf{1}_{2.2}$ and $\mathbf{A} \cdot \mathbf{D} \cdot \mathbf{5}$ is of little surprise (Table 1; also vide infra). However, it might be much more interesting to contrast the behavior of $X B$ donors 2$\mathbf{4}$ with $\mathbf{A}$ and $\mathbf{D}$ to that with $\mathbf{A}$ and $\mathbf{C}$. Especially with $\mathbf{2}$, the isolation of two stoichiometric variations, $\mathbf{A} \cdot \mathbf{D} \cdot \mathbf{2}_{2}$ and
A $\cdot \mathbf{D}_{2} \cdot \mathbf{2}_{2}$, witnesses the delicate interplay of interactions dictating the exact supramolecular outcome.

a)

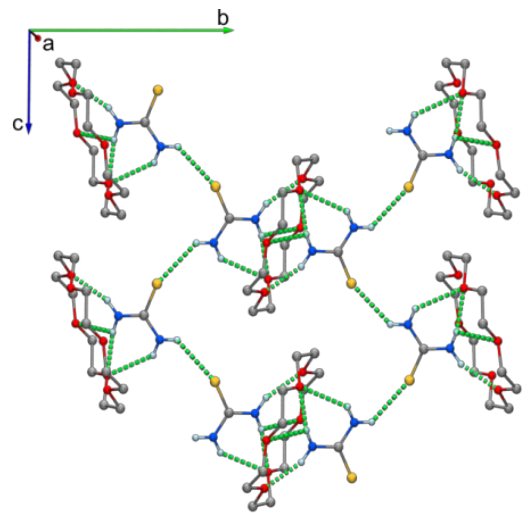

b)

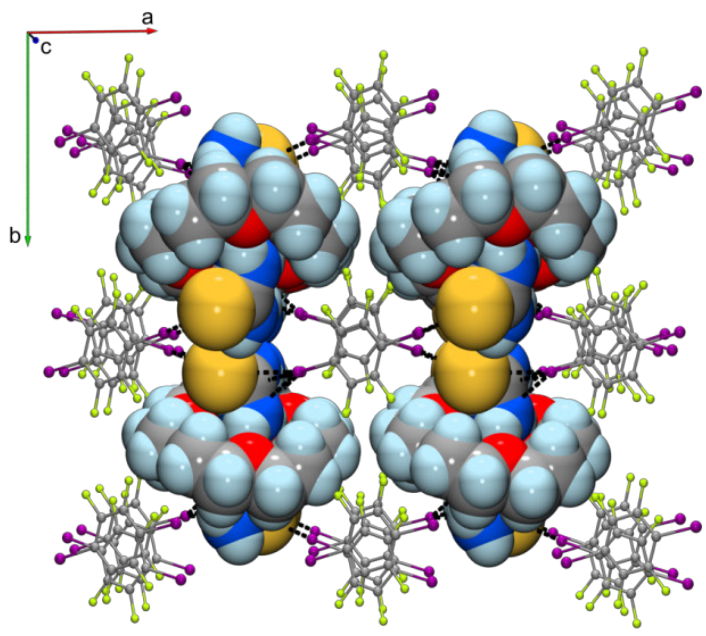

c)

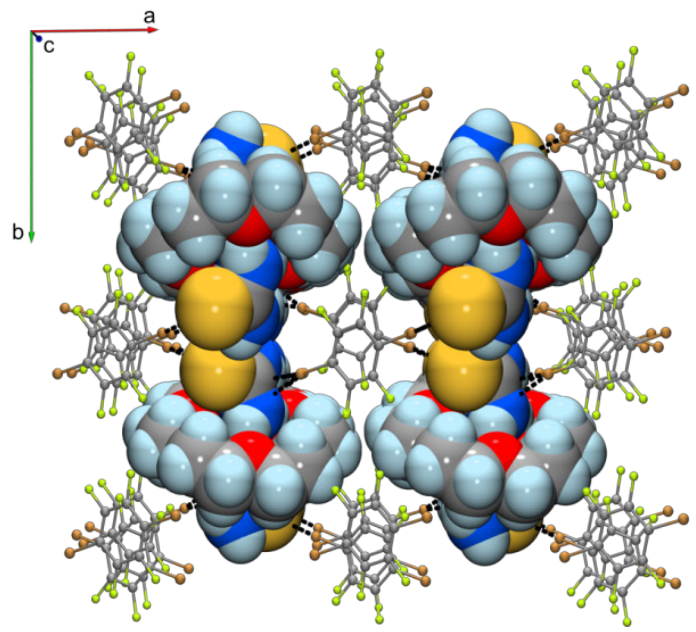

Figure 1. a) Hydrogen bonded $\mathbf{A} \cdot \mathbf{C}_{2} 2 \mathrm{D}$ sheets in $\mathbf{A} \cdot \mathbf{C}_{2} \cdot \mathbf{3}_{2}$ (and $\mathbf{A} \cdot \mathbf{C}_{2} \cdot \mathbf{4}_{2}$ ) with hydrogen atoms of $\mathbf{A}$ omitted for clarity; b) Packing of $\mathbf{A} \cdot \mathbf{C}_{2} \cdot \mathbf{3}_{2}$, with columns of $\mathbf{3}$ between the $\mathbf{A} \cdot \mathbf{C}_{2}$ sheets; C) Packing of $\mathbf{A} \cdot \mathbf{C}_{2} \cdot \mathbf{4}_{2}$, with columns of $\mathbf{4}$ between the $\mathbf{A} \cdot \mathbf{C}_{2}$ sheets. 


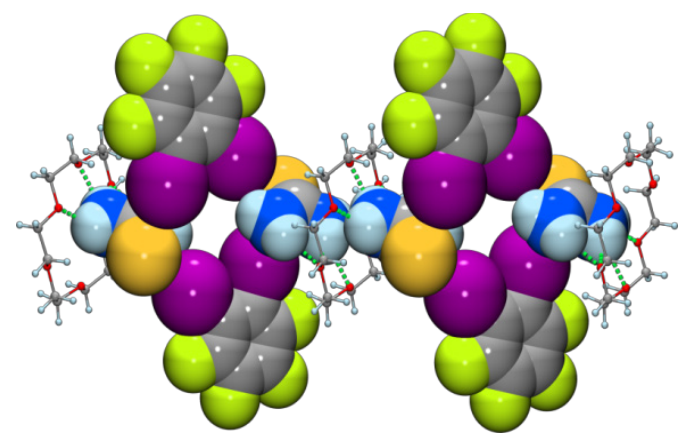

Figure 2. Chains of $\mathbf{A} \cdot \mathbf{C}_{2}$ motif II moieties, formed through $\mathrm{C}-\mathrm{I} \cdots \mathrm{S}$ halogen bonding with 2 as a ditopic donor.

Initially, $\mathbf{A} \cdot \mathbf{D} \cdot \mathbf{2}_{2}$ is formed as the kinetic product, with $\mathbf{A}$ and $\mathbf{D}$ assembling into motif $\mathbf{I}$ and $\mathbf{2}$ only serving as a monotopic halogen bond donor, not utilizing its full potential (Figure 3a). ${ }^{33}$ The system evolves, however, resulting in the formation of $\mathbf{A} \cdot \mathbf{D}_{2} \cdot \mathbf{2}_{2}$ with the re-emerging motif II, and the formation of chains through $\mathrm{N}-\mathrm{H} \cdots \mathrm{S}$ hydrogen bonds supported by $\mathrm{C}-\mathrm{I} \cdots \mathrm{S}$ and $\mathrm{C}-1 \cdots \mathrm{O}$ halogen bonding with 2 again acting as a ditopic donor (Figure 3b).

a)
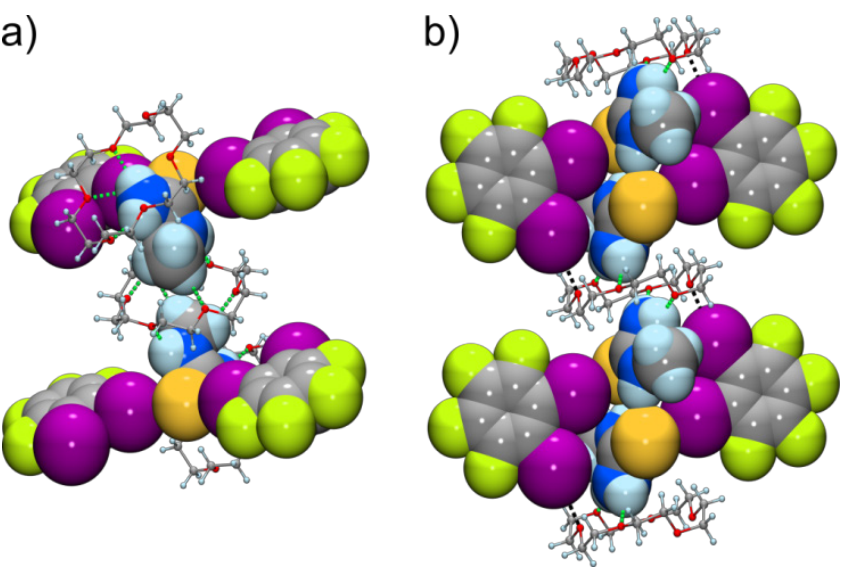

Figure 3. a) Hydrogen bonded motif I formed in $\mathbf{A} \cdot \mathbf{D} \cdot \mathbf{2}_{2}$, decorated by 2 acting as a monotopic halogen bond donor; b) Motif II in $\mathbf{A} \cdot \mathbf{D}_{2} \cdot \mathbf{2}_{2}$, with $\mathbf{A} \cdot \mathbf{D}_{2}$ moieties connected by $\mathrm{N}-\mathrm{H} \cdots \mathrm{S}$ hydrogen bonds and $\mathrm{C}-\mathrm{I} \cdots \mathrm{S}$ halogen bonds with 2 , now as a ditopic donor. Disorder of $\mathbf{2}$ is omitted in a).

Next, the structure of $\mathbf{A} \cdot \mathbf{D}_{2} \cdot \mathbf{3}_{2}$, featuring thiourea $\mathbf{D}$ in the more stable cis conformation ${ }^{40}$ still exhibits motif II, with $\mathbf{A} \cdot \mathbf{D}_{2}$ moieties connected into chains through $C-\mid \cdots S$ halogen bonds with XB donor 3 (Figure 4a). Based on previous observations with $\mathbf{A}$ and $\mathbf{C}$, formation of an isomorphous structure might be expected with 4 . Yet, with the $C-\mid \cdots S$ halogen bonds playing a pivotal role in the structure of $\mathbf{A} \cdot \mathbf{D}_{2} \cdot \mathbf{3}_{2}$, it seems that the presumably weaker XB donor 4 cannot support the formation of an isomorphous structure through $\mathrm{C}-\mathrm{Br} \cdots \mathrm{S}$ halogen bonds. Instead, the balance of interactions is once again tipped in favor of motif $\mathbf{I}$, resulting in $\mathbf{A} \cdot \mathbf{D} \cdot \mathbf{4}_{2}$. While the stoichiometry might be surprising at first, given that $\mathbf{4}$ is a ditopic XB donor, it is a result of each molecule of $\mathbf{4}$ forming one $\mathrm{C}-\mathrm{Br} \cdots \mathrm{S}$ and one $\mathrm{C}-\mathrm{Br} \cdots \mathrm{O}$ halogen bond respectively with $\mathbf{D}$ and $\mathbf{A}$ (Figure $\mathbf{5}$ ). a)

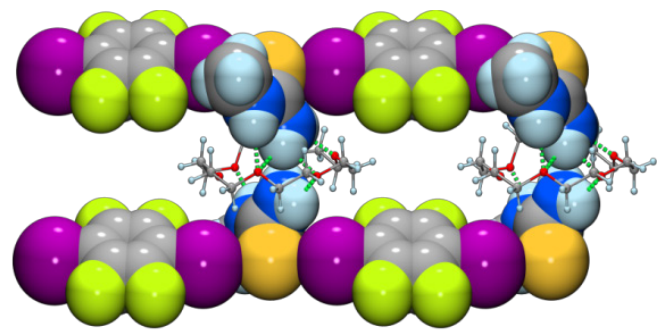

b)

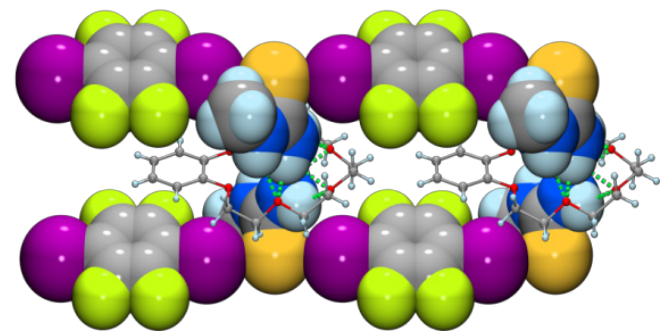

Figure 4. Halogen bonded ribbons in the isomorphous structures of a) $\mathbf{A} \cdot \mathbf{D}_{2} \cdot \mathbf{3}_{2}$ and b) $\mathbf{B} \cdot \mathbf{D}_{2} \cdot \mathbf{3}_{2}$, comprising motif II type $\mathbf{A} \cdot \mathbf{D}_{2} / \mathbf{B} \cdot \mathbf{D}_{2}$ moieties linked by $\mathbf{C}-\mid \cdots S$ halogen bonds with $\mathbf{3}$. Disorder of $\mathbf{A}$ is not shown in $\mathbf{a})$.

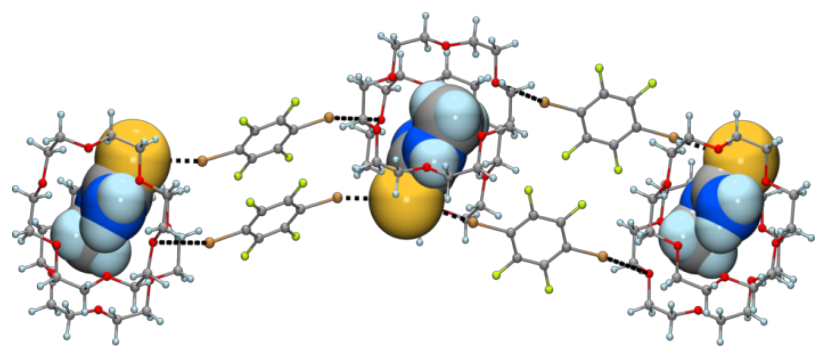

Figure 5. Hydrogen bonded columns (motif I) connected by $\mathrm{C}-\mathrm{Br} \cdots \mathrm{S}$ and $\mathrm{C}-\mathrm{Br} \cdots \mathrm{O}$ halogen bonds with $\mathbf{4}$ in $\mathrm{A} \cdot \mathbf{D} \cdot \mathbf{4}_{2}$.

Remarkably, co-crystallization experiments on the component subset comprising crown ether $\mathbf{A}$, thioureas C and $\mathbf{D}$, and XB donors $\mathbf{1 - 5}$ resulted in a $100 \%$ success rate, with each of the combinations yielding at least one ternary co-crystal. Repeating all these experiments with benzo-crown ether $\mathbf{B}$ instead of $\mathbf{A}$, however, halved the success rate, showing $\mathbf{B}$ to be a much more selective co-former than A. Still, the structures of the ternary cocrystals obtained with such a selective co-former might reveal particularly robust supramolecular motifs, suggesting possible improvements to our synthetic strategy.

A unique example among all the ternary co-crystals presented here, the combination of benzo-crown ether $B$, thiourea $\mathbf{C}$ and $\mathrm{XB}$ donor $\mathbf{1}$ yielded a polymorphic pair of co-crystals $\alpha-B \cdot C \cdot 1_{2}$ (Figure 6a) and $\beta-B \cdot C \cdot 1_{2}$ (Figure $6 \mathrm{~b}$ ), both of which feature the motif III, not observed in any of the other structures.
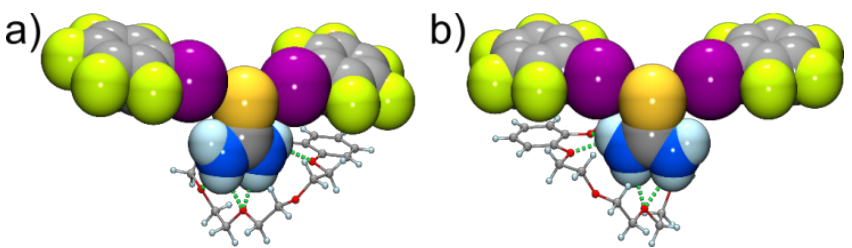

Figure 6. Discrete $\mathbf{B} \cdot \mathbf{C} \cdot \mathbf{1}_{2}$ assemblies in the two polymorphs a) $\alpha-\mathbf{B} \cdot \mathbf{C} \cdot \mathbf{1}_{2}$ and b) $\beta-\mathbf{B} \cdot \mathbf{C} \cdot \mathbf{1}_{2}$. 
The two polymorphs arise from different packing of the discrete $\mathbf{B} \cdot \mathbf{C} \cdot \mathbf{1}_{2}$ moieties: in both structures, they can be seen as forming layers through stacking of the aromatic rings of $\mathbf{B}$ and $\mathbf{1}$. These layers then pack in a parallel $[\beta-$ $\mathbf{B} \cdot \mathbf{C} \cdot \mathbf{1}_{2}$ ] or anti-parallel fashion $\left[\alpha-\mathbf{B} \cdot \mathbf{C} \cdot \mathbf{1}_{2}\right.$ ], giving rise to two polymorphs (Figures S21-S24). To the best of our knowledge, this is the first example of polymorphism in ternary co-crystals. Both of the other two structures with the combination of $\mathbf{B}$ and $\mathbf{C}$, namely $\mathbf{B} \cdot \mathbf{C} \cdot \mathbf{3}$ and $\mathbf{B} \cdot \mathbf{C} \cdot \mathbf{5}$, show the $\mathbf{B}$ and $\mathbf{C}$ forming motif $\mathbf{I}$, with the hydrogen bonded columns B.C interlinked by $\mathrm{C}-1 \cdots \mathrm{S}$ halogen bonds, respectively through 3 (Figures S25, S26b) or 5 (Figure 8c). Surprisingly, the stacking of the phenyl rings of $\mathbf{B}$ and $\mathbf{3}$ does not seem to interfere with the formation of motif I between B and C (Figure S26a) or the infinite halogen-bonded chains between $\mathbf{C}$ and $\mathbf{3}$, with all three seemingly acting in concert (Figure S26b).

Finally, only two ternary co-crystals were observed when the combination of $\mathbf{B}$ and $\mathbf{D}$ was employed. One of the two is $\mathbf{B} \cdot \mathbf{D}_{2} \cdot \mathbf{3}_{2}$, reminiscent of $\mathbf{A} \cdot \mathbf{D}_{2} \cdot \mathbf{3}_{2}$ not only in its stoichiometry but actually forming an isomorphous structure consisting of $\mathbf{B} \cdot \mathbf{D}_{2} \cdot$ motif II-type moieties forming ribbons through $\mathrm{C}-\mathrm{I} \cdots \mathrm{S}$ halogen bonds with 3 (Figure 4b). This might be a unique example of quite a drastic perturbation (replacement of the plain 18-crown-6 A with benzo-substituted $\mathbf{B}$ ) which does not radically influence the overall structure. No less remarkably, like all the cocrystals involving $X B$ donor $\mathbf{5}$ so far, the B.D.5 once again sports the motif $\mathbf{I}$ columns of $\mathbf{B}$ and $\mathbf{D}$ interlinked by halogen bonding with $\mathbf{5}$ (vide infra).

Clearly, two intriguing aspects of the mentioned structures deserve particular highlighting. First, the curious stoichiometry of $\mathbf{A} \cdot \mathbf{D} \cdot \mathbf{1}_{2.2}$ compared to similar $\mathbf{A} \cdot \mathbf{C} \cdot \mathbf{1}_{2}$ merits further explanation. This seems to be a consequence of the elongation of motif I-type columns upon changing from $\mathbf{C}$ to $\mathbf{D}$, which is best quantified through the distance between the centroids of neighboring molecules of $\mathbf{A}$ in the same column (Figure 7). This expansion of the structure is also reflected in the increased distance between molecules of XB donor $\mathbf{1}$ in $\mathbf{A} \cdot \mathbf{D} \cdot \mathbf{1}_{2.2}$ (Figure 7b), allowing for inclusion of an excess of 1 (Figure S10).

Another striking observation is that of the identical 1:1:1 stoichiometry in all ternary co-crystals involving $\mathbf{5}$, with motif I also appearing in all four crystal structures, despite their differences (Figure 8). Specifically, it is interesting to note how the difference in halogen bonding connectivity e.g. between A.C.5 (Figure $\mathbf{8 a}$ ) and A.D.5 (Figure $\mathbf{8 b}$ ) does not affect the above mentioned stoichiometry and the appearance of motif I columns. Moreover, the effect of the motif I column elongation upon change from $\mathbf{C}$ to $\mathbf{D}$ again becomes evident when comparing B.C.5 (Figure 8C) and B.D.5 (Figure 8d), with increased distance allowing for the pairing of $\mathbf{B} \cdot \mathbf{D}$ columns through phenyl ring stacking in $\mathbf{B} \cdot \mathbf{D} \cdot \mathbf{5}$.

Looking at the co-crystal series with XB donors 1 and 5 raises the question: What are the different (possibly shared) characteristics of $\mathbf{1}$ and $\mathbf{5}$ driving the formation a)

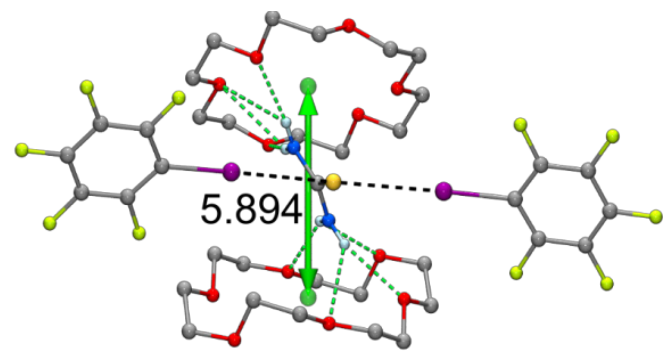

b)

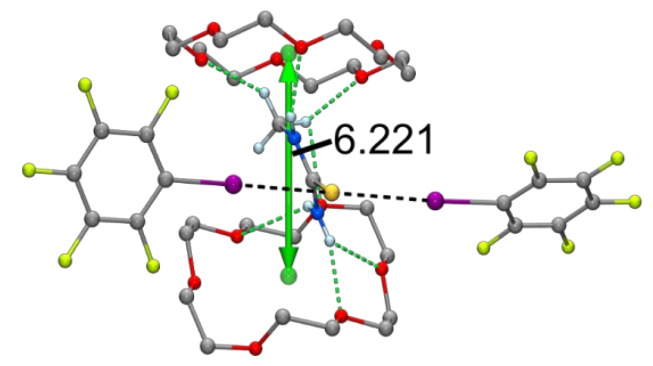

Figure 7. Different metrics of the motif I columns in a) $\mathbf{A} \cdot \mathbf{C} \cdot \mathbf{1}_{2}$ and b) $\mathbf{A} \cdot \mathbf{D} \cdot \mathbf{1}_{2.2}$. Hydrogen and halogen bonds are shown as dashed green and black lines, respectively, while the hydrogen atoms of $\mathbf{A}$ are omitted for clarity.

of motif I (or motif III, still with the same stoichiometry), unlike the motif II formed with 2, $\mathbf{3}$ and $\mathbf{4}$ as halogen bond donors. In fact, it seems that XB donors 1 and 5 having little in common with 2-4 might just be at the root of their different behavior: While all of 2-4 are aromatic and ditopic donors, $\mathbf{1}$ is aromatic but monotopic, and $\mathbf{5}$ is ditopic but aliphatic. Both the monotopic 1 and the conformationally flexible $\mathbf{5}$ are expected to eliminate or reduce the communication between the halogen bond acceptors, with any significant packing preferences also being absent in the case of $\mathbf{5}$. This in turn seems to allow for the almost perfect orthogonality of hydrogen and halogen bonding in these systems, reliably yielding ternary co-crystals with expected stoichiometries and supramolecular motifs.

On the other hand, focusing the analysis on the halogen bonding reveals very few surprises and only a few general structural features are worth commenting. Both thioureas $\mathbf{C}$ and $\mathbf{D}$ seem to act almost exclusively as bifurcated acceptors, ${ }^{41}$ with the only exception being $\mathbf{A} \cdot \mathbf{D}_{2} \cdot \mathbf{2}_{2}$ (Figure $\mathbf{3 b}$ ). The relative geometries manifest a notable degree of variation, judged especially by the $X \cdots S \cdots X$ and $X \cdots S-C$ angles (Tables S3, S5, ..., S35). While the former can be explained by a relatively weak interaction energy dependence on the $X \cdots S$ distance and the $\mathrm{N}-\mathrm{C}-\mathrm{S} \cdots \mathrm{X}$ torsional angle on the relevant intervals, as established by Pennington and co-workers, ${ }^{33}$ the latter can be explained by a partial $\mathrm{C}-\mathrm{X} \cdots \pi$ character due to the $\pi(C=S)$ electron participation. Moreover, the quadrupolar stacking of the aromatic rings also influences the orientation of the aromatic halogen bond donor with respect to the thiourea acceptor, such as in $\alpha / \beta$ B.C.1 $1_{2}$ (Figure 6). Nevertheless, the halogen bonding seems to operate rather predictably especially with 1,3 and 5 as donors, forming discrete $1 \cdots \mathbf{C} / \mathbf{D} \cdots \mathbf{1}$ assemblies or infinite $\cdots C / D \cdots 3 / 5 \cdots C / D \cdots 3 / 5 \cdots$ chains. 
a)

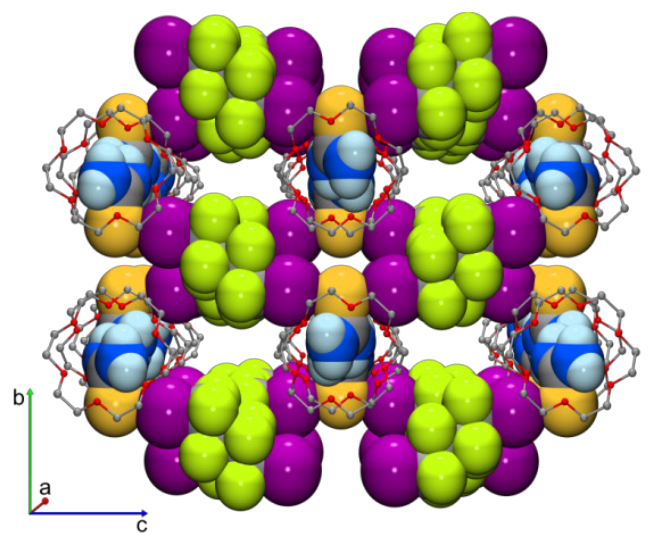

b)

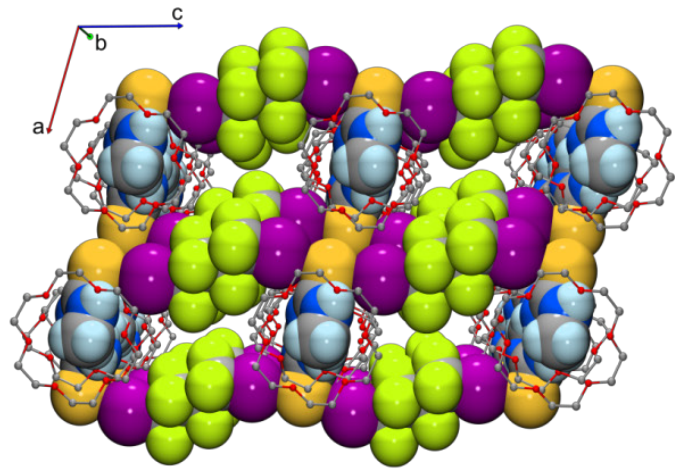

c)

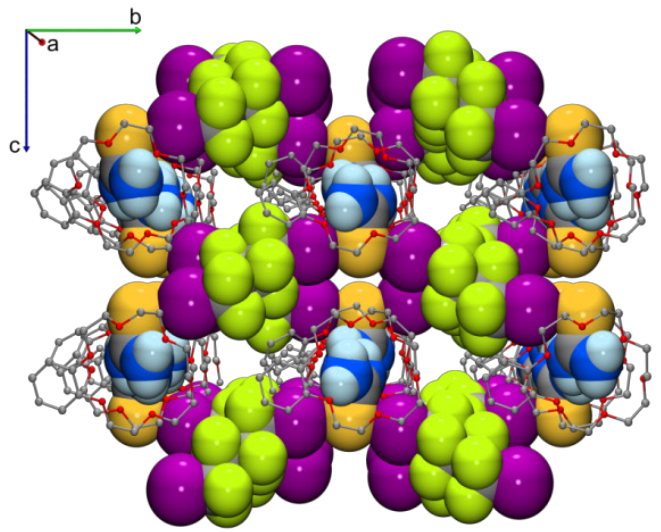

d)

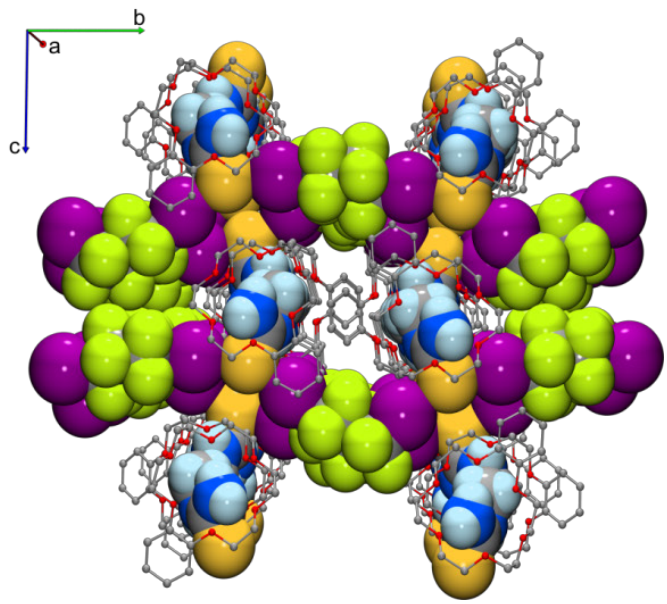

Figure 8. Linking of the hydrogen bonded motif I columns by halogen bonds with $\mathbf{5}$ in the structures of a) A.C.5, b) A.D.5, C) B.C.5 and d) B.D.5. Hydrogen atoms of $\mathbf{A}$ and $\mathbf{B}$ and minor disorder components are omitted for clarity.

In contrast, the sterical crowding of the donor iodine atoms in ortho-positions of $\mathbf{2}$ seems to induce some peculiarity in its behavior, causing it to act as a monotopic donor in $\mathbf{A} \cdot \mathbf{D} \cdot \mathbf{2}_{2}$ (Figure $\mathbf{3 a}$ ), to form one $C-\mid \cdots S$ and one $C-\mid \cdots O$ halogen bond in $\mathbf{A} \cdot \mathbf{D}_{2} \cdot \mathbf{2}_{2}$, reinforcing the hydrogen bonded chains (Figure $\mathbf{3 b}$ ), only to emerge as the seemingly key interaction in linking individual $\mathbf{A} \cdot \mathbf{C}_{2}$ moieties into chains in $\mathbf{A} \cdot \mathbf{C}_{2} \cdot \mathbf{2}_{2}$ (Figure 2). Similarly, the expectedly weaker $\mathrm{C}-\mathrm{Br} \cdots \mathrm{S}$ halogen bonding seems to fall victim to aromatic ring stacking of $\mathbf{4}$ in $\mathbf{A} \cdot \mathbf{C}_{2} \cdot \mathbf{4}_{2}$, with one of the two symmetrically independent $\mathrm{C}-\mathrm{Br} \cdots \mathrm{S}$ halogen bonds assuming exclusively $\mathrm{C}-\mathrm{Br} \cdots \pi$ character (Figure S6), or to the realization of the robust hydrogen bonding motif I and stacking of $\mathbf{4}$ in $\mathbf{A} \cdot \mathbf{D} \cdot \mathbf{4}_{2}$, forming one $\mathrm{C}-\mathrm{Br} \cdots \mathrm{S}$ and one $\mathrm{C}-\mathrm{Br} \cdots \mathrm{O}$ halogen bond instead (Figure 5), reminiscent of $\mathbf{2}$ in $\mathbf{A} \cdot \mathbf{D}_{2} \cdot \mathbf{2}_{2}$.

\section{SUMMARY AND CONCLUSIONS}

Our work outlines a design-based strategy for construction of ternary co-crystals based on the ability of thioureas to simultaneously engage in hydrogen and halogen bonding in an orthogonal manner, with the crown ethers and the perfluorocarbon halides as the other co-formers. This ability is presumed to arise from the fundamentally different nature of the two interactions, the predominantly electrostatic hydrogen bonding and the halogen bonding with a significant charge-transfer character. The strategy was tested on a compact but diverse set of commercially available components, yielding ternary co-crystals for $15 / 20$ different component combinations. Detailed structural analysis was undertaken in order to identify and critically examine the variables influencing its efficacy.

The hydrogen bonding in the obtained ternary cocrystal structures is classified as belonging to one of three different motifs (I, II and III), whose formation can be correlated with the particular structural features of the components. Namely, the stacking of the aromatic rings (present in benzo-crown ether B and XB donors 1-4), sterical crowding of halogen bond donor atoms (as in e.g. 2) or the Br-based donor (4) all seem to be important variables which can influence the co-crystal formation. On the other hand, crown ethers $\mathbf{A}$ and $\mathbf{B}$ as well as thioureas $\mathbf{C}$ and $\mathbf{D}$ are shown to be structurally equivalent to a great degree, with the structural features often preserved on changing between $\mathbf{A}$ and $\mathbf{B}$ (e.g. for $\mathbf{A} \cdot \mathbf{D}_{2} \cdot \mathbf{3}_{2} / \mathbf{B} \cdot \mathbf{D}_{2} \cdot \mathbf{3}_{2}$ ) or $\mathbf{C}$ and $\mathbf{D}$ (e.g. for $\mathbf{A} \cdot \mathbf{C} \cdot \mathbf{1}_{2} / \mathbf{A} \cdot \mathbf{D} \cdot \mathbf{1}_{2.2}$ ). Remarkably, all of these observations are validated by co-crystals with the non-aromatic, linear, flexible and iodine-based XB donor 5, which all exhibit the same hydrogen bonding motif $I$ and the same halogen bonded infinite chains.

Finally, the presented results show how the orthogonality of hydrogen and halogen bonding can be achieved and employed to prepare complex molecular solids ternary co-crystals. The principles outlined here hold great potential for the effective design of highly complex, functional molecular solids from simple building blocks. This research direction, as well as a detailed study of the particular interactions and their interplay in these systems, is currently being pursued in our laboratory.

\section{ASSOCIATED CONTENT}


Supporting Information. Experimental details, powder diffraction studies, single crystal $X$-ray structure analysis with additional figures and tables of selected hydrogen and halogen bonds. This material is available free of charge via the Internet at http://pubs.acs.org. CCDC 1469004-1469024 contain the supplementary crystallographic data for this paper. The data can be obtained free of charge from The Cambridge Crystallographic Data Centre via www.ccdc.cam.ac.uk/structures.

\section{AUTHOR INFORMATION}

\section{Corresponding Author}

*kari.t.rissanen@jyu.fi

\section{Notes}

The authors declare no competing financial interest.

\section{ACKNOWLEDGMENTS}

F.T. and K.R. gratefully acknowledge the Academy of Finland (K.R. grant nos. 263256 and 265328) and the University of Jyväskylä for financial support. F.T. acknowledges the PhD scholarship from NGS-NANO and the 2015 Ludo Frevel Crystallography Scholarship from ICDD.

\section{REFERENCES}

1. Bond, A. D. CrystEngComm 2007, 9, 833-834.

2. Aakeröy, C. B. Acta Cryst. 2015, B71, 387-391.

3. Stahly, G. P. Cryst. Growth Des. 2009, 9, 4212-4229.

4. Almarsson, O.; Zaworotko, M. J. Chem. Commun. 2004, 1889-1896.

5. Schultheiss, N.; Newman, A. Cryst. Growth Des. 2009, 9, 2950-2967.

6. Friščić, T.; Jones, W. J. Pharm. Pharmacol. 2010, 62, 1547-1559.

7. Yan, D.; Delori, A.; Lloyd, G. O.; Friščić, T.; Day, G. M.; Jones, W.; Lu, J.; Wei, M.; Evans, D. G.; Duan, X. Angew. Chem. Int. Ed. 2011, 50, 12483-12486.

8. Bushuyev, O. S.; Corkery, T. C.; Barrett, C. J.; Friščić, T. Chem. Sci. 2014, 5, 3158-3164.

9. Tayi, A. S.; Shveyd, A. K.; Sue, A. C.-H.; Szarko, J. M.; Rolczynski, B. S.; Cao, D.; Kennedy, T. J.; Sarjeant, A. A.; Stern, C. L.; Paxton, W. F.; Wu, W.; Dey, S. K.; Fahrenbach, A. C.; Guest, J. R.; Mohseni, H.; Chen, L. X.; Wang, K. L.; Stoddart, J. F.; Stupp, S. I. Nature 2012, 488, 485-489.

10. Bolton, O.; Matzger, A. J. Angew. Chem. Int. Ed. 2011, 50, 8960-8963.

11. Aakeröy, C. B.; Wijethunga, T. K.; Desper, J. Chem. Eur. J. 2015, 21, 11029-11037.

12. Aakeröy, C. B.; Beatty, A. M.; Helfrich, B. A. Angew. Chem. Int. Ed. 2001, 113, 3340-3342.

13. Etter, M. C. Acc. Chem. Res. 1990, 23, 120-126.

14. Desiraju, G. R. Angew. Chem. Int. Ed. 1995, 34, 23112327.

15. Aakeröy, C. B.; Desper, J.; Urbina, J. F. Chem. Commun. 2005, 2820-2822.

16. Aakeröy, C. B.; Desper, J.; Smith, M. M. Chem. Commun. 2007, 3936-3938.

17. Adsmond, D. A.; Sinha, A. S.; Khandavilli, U. B. R.; Maguire, A. R.; Lawrence, S. E. Cryst. Growth Des. 2016, 16, 59-69.

18. Tothadi, S.; Mukherjee, A.; Desiraju, G. R. Chem. Commun. 2011, 47, 12080-12082.

19. Dubey, R.; Desiraju, G. R. Angew. Chem. Int. Ed. 2014, 53, 13178-13182.

20. Mir, N. A.; Dubey, R.; Tothadi, S.; Desiraju, G. R. CrystEngComm 2015, 17, 7866-7869.

21. Tothadi, S.; Desiraju, G. R. Chem. Commun. 2013, 49, 7791-7793.
22. Tothadi, S.; Sanphui, P.; Desiraju, G. R. Cryst. Growth Des. 2014, 14, 5293-5302.

23. Mir, N. A.; Dubey, R.; Desiraju, G. R. IUCrJ 2016, 3, 96101.

24. Dubey, R.; Mir, N. A.; Desiraju, G. R. IUCrJ 2016, 3, 102107.

25. Smolka, T.; Boese, R.; Sustmann, R. Struct. Chem. 1999, 10, 429-431.

26. Shivanyuk, A.; Spaniol, T.; Rissanen, K.; Kolehmainen, E.; Böhmer, V. Angew. Chem. Int. Ed. 2000, 39, 3497-3500.

27. Beyeh, N. K.; Cetina, M.; Löfman, M.; Luostarinen, M.; Shivanyuk, A.; Rissanen, K. Supramol. Chem. 2010, 22, 737750.

28. Beyeh, N. K.; Cetina, M.; Rissanen, K. Chem. Commun. 2014, 50, 1959-1961.

29. Beyeh, N. K.; Valkonen, A.; Bhowmik, S.; Pan, F.; Rissanen, K. Org. Chem. Front. 2015, 2, 340-345.

30. Beyeh, N. K.; Pan, F.; Rissanen, K. Angew. Chem. Int. Ed. 2015, 54, 7303-7307.

31. Pan, F.; Beyeh, N. K.; Rissanen, K. J. Am. Chem. Soc. 2015, 137, 10406-10413.

32. Aakeröy, C. B.; Beatty, A. M.; Helfrich, B. A. J. Am. Chem. Soc. 2002, 124, 14425-14432.

33. Arman, H. D.; Gieseking, R. L.; Hanks, T. W.; Pennington, W. T. Chem. Commun. 2010, 46, 1854-1856.

34. Robertson, C. C.; Perutz, R. N.; Brammer, L.; Hunter, C. A. Chem. Sci. 2014, 5, 4179-4183.

35. Drew, M. G. B.; Nicholson, D. G. Acta Cryst. 1985, C41, 1358-1360.

36. Watson, W. H.; Galloy, J.; Grossie, D. A.; Voegtle, F.; Mueller, W. M. J. Org. Chem. 1984, 49, 347-353.

37. Wishkerman, S.; Bernstein, J.; Hickey, M. B. Cryst. Growth Des. 2009, 9, 3204-3210.

38. Wang, H.; Wang, W.; Jin, W. J. $\sigma$-Hole Bond vs $\pi-H o l e$ Bond: A Comparison Based on Halogen Bond Chem. Rev. [Online early access]. 10.1021/acs.chemrev.5b00527. Published Online: Feb 17, 2016. http://pubs.acs.org/doi/abs/10.1021/acs.chemrev.5b00527 (accessed Mar 15, 2016).

39. Laurence, C.; Graton, J.; Berthelot, M.; El Ghomari, M. J. Chem. Eur. J. 2011, 17, 10431-10444.

40. Bryantsev, V. S.; Hay, B. P. J. Phys. Chem. A 2006, 110, 4678-4688.

41. Cinčić, D.; Friščić, T.; Jones, W. CrystEngComm 2011, 13, 3224-3231. 
SYNOPSIS TOC (Word Style "SN_Synopsis_TOC"). If you are submitting your paper to a journal that requires a synopsis graphic and/or synopsis paragraph, see the Instructions for Authors on the journal's homepage for a description of what needs to be provided and for the size requirements of the artwork.

\section{For Table of Contents Only}

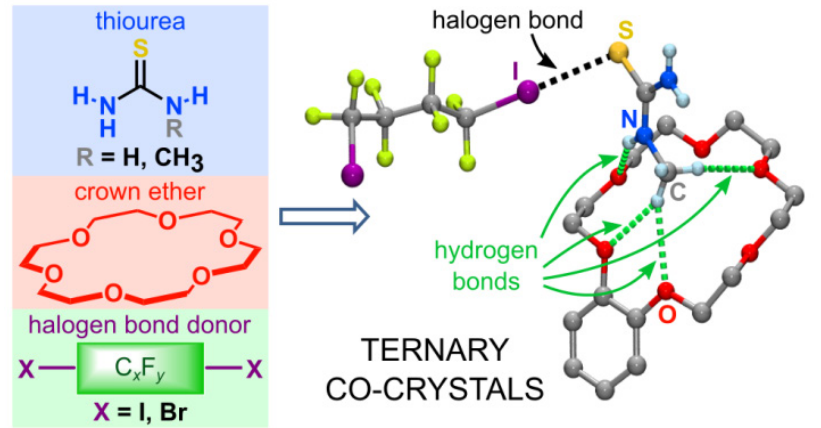

